\title{
Modified dose of melphalan-prednisone in multiple myeloma patients receiving bortezomib plus melphalan-prednisone treatment
}

\author{
Se Ryeon Lee, Hojoon Choi, Byung Hyun Lee, Ka-Won Kang, Eun Sang Yu, Dae Sik Kim, Yong Park, \\ Chul Won Choi, Byung Soo Kim, and Hwa Jung Sung
}

Division of Hematology and Oncology, Department of Internal Medicine, Korea University College of Medicine, Seoul, Korea
Received: April 18, 2018

Revised : May 8, 2018

Accepted: May 9, 2018

\section{Correspondence to}

Hwa Jung Sung, M.D.

Division of Hematology and

Oncology, Department of Internal

Medicine, Korea University Ansan

Hospital, 123 Jeokgeum-ro, Dan-

won-gu, Ansan 15355, Korea

Tel: $+82-31-412-6549$

Fax: +82-31-412-5984

E-mail: drhwajung@gmail.com
Background/Aims: Bortezomib plus melphalan-prednisone (VMP) is a standard treatment for multiple myeloma, particularly for patients who are ineligible for high-dose therapy. However, early discontinuation or treatment modification is often needed owing to adverse events. The aim of this study was to investigate the clinical outcomes of modifying the dose of melphalan-prednisone (MP) in patients receiving VMP.

Methods: We examined 67 patients who received a modified dose of MP, and 38 patients who received the regularly planned dose of MP. We then analyzed clinical differences between the groups.

Results: Although there was no difference in the proportion of discontinuation due to adverse events between dose groups, more patients in the planned-dose group experienced earlier discontinuation in general. The overall response rate (ORR) was $81.0 \%$ and complete response (CR) rate was 30.5\%. After a median 15.7 months of follow-up, the median progression-free survival (PFS) and overall sur$\operatorname{vival}(\mathrm{OS})$ were 25.0 and 47.8 months, respectively. There was no significant difference in the ORR, CR, PFS, and OS of the two dose groups. A median of four cycles were delivered, and the median cumulative bortezomib dose was $41.6 \mathrm{mg} / \mathrm{m}^{2}$. The median PFS in patients with doses $\geq 41.6 \mathrm{mg} / \mathrm{m}^{2}$ was longer than that in patients with doses $<41.6 \mathrm{mg} / \mathrm{m}^{2}$ (35.1 months vs. 9.6 months). However, when MP was < $50 \%$ of the planned dose, PFS and OS were poor.

Conclusions: Modifying the dose of MP might be a feasible and effective therapeutic approach for multiple myeloma patients receiving VMP treatment.

Keywords: Multiple myeloma; Bortezomib; Melphalan; Prednisone; Modified

\section{INTRODUCTION}

Multiple myeloma (MM) is a malignant neoplasm of plasma cells derived from a single clonal expansion in the bone marrow (BM) and is characterized by bone destruction, renal failure, anemia, and hypercalcemia [1]. During the past decade, high-dose therapy with he- matopoietic stem cell transplantation has become the preferred treatment for patients under the age of 65 years old, but older patients and patients with clinically significant comorbidities generally cannot tolerate this treatment. Since the median age at diagnosis of myeloma is approximately 70 years, more than half of the patients with newly diagnosed myeloma may not be 
eligible for high-dose therapy. The proteasome inhibitor bortezomib is reported to be an active agent against relapsed or refractory MM $[2,3]$. An international phase III Velcade as Initial Standard Therapy in Multiple Myeloma (VISTA) trial demonstrated that bortezomib plus melphalan-prednisone (VMP) was superior to melphalan-prednisone (MP) in all efficacy measurements, including time to disease progression, response rates, and overall survival (OS), in previously untreated patients with newly diagnosed MM in the USA $[4,5]$. On the basis of the results of the VISTA study, VMP is now recognized as a standard treatment for MM in patients $\geq 65$ years of age. In Korea, VMP is the most commonly used regimen for treating MM patients who are ineligible for high-dose therapy. However, it is difficult to adhere rigidly to VMP therapy with elderly MM patients because they often have impaired BM function, altered drug metabolism, and the presence of comorbid diseases, all of which could lead to adverse events during treatment. Currently there are no appropriate guidelines regarding whether or how doses of MP or bortezomib should be reduced when adverse events occur during VMP treatment. Recently, some studies have indicated that a higher cumulative dose of bortezomib can lead to improved OS [6,7]; however, there are no comparable data regarding the dose intensity of MP. The aim of this study was therefore to investigate the clinical outcomes of modifying the MP dose in MM patients compared with the regularly planned MP dose from the beginning of VMP treatment.

\section{METHODS}

Patients who were newly diagnosed with symptomatic MM between January 2011 and January 2018 at three tertiary hospitals (Korea University Anam Hospital, Korea University Guro Hospital, and Korea University Ansan Hospital) of the Korea University Medical Center were analyzed retrospectively. The study was approved by the Institutional Review Board of Korea University Hospital (2018ASo111). All patients provided written informed consent. At the time of diagnosis, none of the patients were candidates for high-dose therapy plus stem cell transplantation because of their age ( $\geq 65$ years old) or coexisting conditions. These patients were treated with
VMP following the approved regimen, which comprised nine 6-week cycles of treatment with melphalan (at a dose of $9 \mathrm{mg} / \mathrm{m}^{2}$ ) and prednisone (at a dose of $60 \mathrm{mg} / \mathrm{m}^{2}$ ) on days 1 to 4 , in combination with bortezomib (at a dose of $1.3 \mathrm{mg} / \mathrm{m}^{2}$ ) on days $1,4,8,11,22,25,29$, and 32 during cycles 1 to 4 , and on days $1,8,22$, and 29 during cycles 5 to 9 . Among the 105 patients examined, 67 (63.8\%) received a modified dose of MP from the first treatment cycle onward (i.e., the reduced-dose group), whereas the remaining 38 patients $(36.2 \%)$ received the regularly planned dose of MP (i.e., the planned-dose group). In the reduced-dose group, MP dose was modified from the planned dose (100\%) to $75 \%$ or $50 \%$ of the planned dose based on the investigator's discretion. This was mainly determined by poor general condition, low body weight, poor nutritional status, and impaired renal function. The modified doses of melphalan administered were $75 \%$ of the planned dose in 46 patients $(68.7 \%)$ and $50 \%$ of the planned dose in 19 patients (28.4\%). Two patients (3.0\%) did not receive melphalan due to impaired renal function. The modified doses of prednisone administered were $75 \%$ of the planned dose in 38 patients (56.7\%) and $50 \%$ of the planned dose in eight patients (11.9\%). Twenty-one patients (31.3\%) received the planned dose of prednisone. All patients in this study were treated with the planned dose of bortezomib (at $1.3 \mathrm{mg} / \mathrm{m}^{2}$ ) from the first cycle onward, whereas only one patient with heart failure in the reduced-dose group was started with a reduced dose of bortezomib (at a dose of $1.0 \mathrm{mg} / \mathrm{m}^{2}$ ). All patients received oral acyclovir for prophylaxis of herpes zoster. Since 2012, the typical method used to administer bortezomib has been switched from IV to subcutaneous injection. The dose, administration interval, and total number of cycles of VMP treatment were also modified based on the investigator's discretion during the treatment. Bortezomib-associated neuropathic pain and peripheral sensory neuropathy were managed by following the established dose-modification guidelines [8]. Patients with myeloma-associated bone disease received bisphosphonates, unless such therapy was contraindicated.

Prior to receiving VMP treatment, all patients were screened by age, sex, Eastern Cooperative Oncology Group performance status (ECOG PS), and for underlying diseases. Clinical features at the time of diagnosis were also analyzed, including levels of serum and 
24-hour urine M-protein, free light chains ( $\kappa$ and $\lambda$ ), percentage of BM plasma cells, the presence of osteolytic lesions, and hemodialysis. Baseline laboratory evaluations, including those of hemoglobin level, absolute neutrophil count and platelet count, serum albumin, serum $\beta_{2}$-microglobulin, serum calcium, serum creatinine, C-reactive protein, and serum lactate dehydrogenase, were performed to evaluate each patient's pre-chemotherapy status and risk. Cytogenetic analyses of BM specimens were performed using conventional cytogenetic protocols and interphase fluorescence in situ hybridization (FISH). Since 2008, the FISH panel for MM in our institute has included tests for immunoglobin heavy chain (14932) break apart, translocation of chromosomes 4 and 14 [t(4;14)], translocation of chromosomes 14 and 16 [t(14;16)], deletion of 13q14, and deletion of 17p13. According to the International Myeloma Working Group (IMWG) 2014 consensus criteria, we considered cytogenetically detected 17p deletion and chromosome 14 translocation to indicate high risk. In FISH analysis, $\mathrm{t}(4 ; 14), \mathrm{t}(14 ; 16)$, and del(17p13) were considered high-risk cytogenetic measures $[9,10]$. Response and progression were assessed by investigators according to the international uniform response criteria for MM [11]. Efficacy and safety were evaluated for all patients who had received at least one dose of bortezomib. The severity of adverse events was evaluated according to version 4.0 of the National Cancer Institute's (NCI) Common Terminology Criteria for Adverse Events.

\section{Statistical analyses}

Patient characteristics and baseline data, as well as effectiveness data, were summarized using descriptive statistics. We also analyzed whether there were differences in efficacy, safety, and survival outcomes due to MP dose reduction in VMP treatment. Both the chi-square test and Fisher's exact test were used to test for significant differences among categorical indices of clinical characteristics, and the Mann-Whitney $U$ test was used to test differences in continuous parameters. Progression-free survival (PFS) was defined as the time interval from the date of diagnosis to the date of observed disease progression. OS was defined as the time interval from first administration of bortezomib to the date of death. Duration of response (DOR) was defined as the time interval from the date of achievement of a partial response to treatment until the date of observed disease progression, relapse, or death from any cause. PFS, OS, and DOR were calculated using the Kaplan-Meier method. Univariate and multivariate analyses of prognostic factors for PFS and OS were performed using the logrank test and Cox proportional hazard method. Analyses were performed using IBM SPSS statistical software version 20.o (IBM Co., Armonk, NY, USA). Statistical significance was defined as a $p$ value of $<0.05$.

\section{RESULTS}

\section{Patient characteristics}

A total of 105 patients newly diagnosed with symptomatic MM, who were not candidates for high-dose therapy plus stem cell transplantation, were evaluated for this study. Table 1 shows the patients' baseline characteristics. The median age of all patients at the time of diagnosis was 70 years old (range, 60 to 85 ), and $27(25.7 \%)$ were $\geq 75$ years of age. Among the patients, 54 (54.0\%) had International Staging System (ISS) stage III myeloma and $89(84.8 \%)$ had comorbidities, including hypertension (53.3\%), diabetes mellitus (20.0\%), renal disease (16.2\%), cardiac disease (12.4\%), and others (47.6\%). In total, 48 patients $(45.7 \%)$ had a serum $\beta_{2}$-microglobulin level of $\geq$ $5.5 \mathrm{mg} / \mathrm{L}$, and $75(71.4 \%)$ had a glomerular filtration rate $<60 \mathrm{~mL} / \mathrm{min}$. Initially, seven patients $(6.7 \%)$ received a weekly schedule of bortezomib treatment from the first cycle onwards, whereas 46 patients $(43.8 \%)$ were changed from a twice-weekly to a weekly administration schedule of bortezomib after the first cycle. In addition, doses of the bortezomib and MP were changed, depending on the patient's condition during the treatment period. Bortezomib dose was reduced to $1.0 \mathrm{mg} / \mathrm{m}^{2}$ and the MP dose was further reduced by $25 \%$ from the initial dose. The manner of reducing the doses of bortezomib and MP was the same for both dose groups. Of the 105 patients, 32 (30.5\%) completed nine cycles of VMP therapy, and nine $(8.6 \%)$ have been receiving the VMP therapy until the present day. Among the 64 patients (60.9\%) for whom the therapy was terminated before the end of nine cycles, the most common reason for termination was adverse events in 26 patients, which included five deaths (unrelated to MM in two patients), disease progression in 18 patients, caregiver's choice in 13 patients, 
Table 1. The baseline characteristics of patients in this study

\begin{tabular}{|c|c|c|c|c|}
\hline \multirow[b]{2}{*}{ Category } & \multirow[b]{2}{*}{ Total $(\mathrm{n}=105)$} & \multicolumn{3}{|c|}{ Treatment group by dose of MP } \\
\hline & & $\begin{array}{l}\text { The reduced-dose group } \\
\qquad(\mathrm{n}=67)\end{array}$ & $\begin{array}{l}\text { The planned-dose group } \\
\qquad(\mathrm{n}=38)\end{array}$ & $p$ value \\
\hline Age, yr & $70(60-85)$ & $70.3(65-85)$ & $68.8(60-79)$ & $0.160^{\mathrm{a}}$ \\
\hline$\geq 75$ & $27(25 \cdot 7)$ & $16(23.9)$ & $11(28.9)$ & $0.568^{b}$ \\
\hline Sex, male/female & $59(56.2) / 46(43.8)$ & $37(55.2) / 30(44.8)$ & $22(57.9) / 16(42.1)$ & $0.791^{b}$ \\
\hline BSA & $1.60\left(1.25^{-2.10}\right)$ & $1.60(1.25-1.86)$ & $1.59\left(1.35^{-2.10}\right)$ & $0.332^{\mathrm{a}}$ \\
\hline ECOG PS & & & & $0.278^{\mathrm{b}}$ \\
\hline 0 & $12(11.4)$ & $6(9.0)$ & $6(15.8)$ & \\
\hline 1 & $74(70.5)$ & $45(67.2)$ & $29(76.3)$ & \\
\hline 2 & $16(15 \cdot 2)$ & $13(19.4)$ & $3(7 \cdot 9)$ & \\
\hline 3 & $2(1.9)$ & $2(3.0)$ & o & \\
\hline 4 & $1(1.0)$ & $1(1.5)$ & 0 & \\
\hline Type of myeloma & & & & $0.246^{b}$ \\
\hline $\operatorname{IgG}$ & $64(61.0)$ & $38(56.7)$ & $26(68.4)$ & \\
\hline $\operatorname{IgA}$ & $23(21.9)$ & $16(23.9)$ & $7(18.4)$ & \\
\hline IgD & $1(1.0)$ & $1(1.5)$ & 0 & \\
\hline $\operatorname{IgM}$ & $1(1.0)$ & $\mathrm{O}$ & $1(2.6)$ & \\
\hline Light chain & $15(14 \cdot 3)$ & $12(17 \cdot 9)$ & $3(7 \cdot 9)$ & \\
\hline Non-secretory & $1(1.0)$ & 0 & $1(2.6)$ & \\
\hline Bone lesions & & & & $0.057^{b}$ \\
\hline None & $19(18.1)$ & $9(13.4)$ & $10(26.3)$ & \\
\hline 1 or 2 lesions & $68(64.8)$ & $49(73.1)$ & $19(50.5)$ & \\
\hline$\geq 3$ lesions & $18(17.1)$ & $9(13.4)$ & $9(23.7)$ & \\
\hline Serum albumin, g/dL & $3 \cdot 3(1.0-4.7)$ & $3.3(1.0-4.7)$ & $3.4(1.9-4.4)$ & $0.860^{\mathrm{a}}$ \\
\hline$<3.5$ & $62(59.0)$ & $41(61.2)$ & $21(55 \cdot 3)$ & $0.553^{\mathrm{b}}$ \\
\hline$\geq 3.5$ & $43(41.0)$ & $26(38.8)$ & $17(44.7)$ & \\
\hline Serum $\beta_{2}-\mathrm{MG}, \mathrm{mg} / \mathrm{L}$ & $5.29(1.10-96.00)$ & $6.15(1.10-96.00)$ & $4.91(1.18-26.00)$ & $0.104^{\mathrm{a}}$ \\
\hline$<2.5$ & $9(8.6)$ & $5(7 \cdot 5)$ & $4(10.5)$ & $0.186^{\mathrm{b}}$ \\
\hline $2.5-5 \cdot 5$ & $46(43.8)$ & $25(37 \cdot 3)$ & $21(55 \cdot 3)$ & \\
\hline$\geq 5 \cdot 5$ & $48(45 \cdot 7)$ & $36(53.7)$ & $12(31.6)$ & \\
\hline $\mathrm{NE}$ & $2(1.9)$ & $1(1.5)$ & $1(2.6)$ & \\
\hline ISS stage & & & & $0.371^{b}$ \\
\hline I & $10(9 \cdot 5)$ & $4(6.0)$ & $6(15 \cdot 7)$ & \\
\hline II & $39(37.1)$ & $27(40.3)$ & $12(31.6)$ & \\
\hline III & $54(54.0)$ & $35(52.2)$ & $19(50.0)$ & \\
\hline $\mathrm{NE}$ & $2(1.9)$ & $1(1.5)$ & $1(2.6)$ & \\
\hline Risk group $^{c}$ & & & & $0.062^{b}$ \\
\hline High risk & $22(21.0)$ & $11(16.4)$ & $11(28.9)$ & \\
\hline Standard risk & $77(73 \cdot 3)$ & $54(80.6)$ & $23(60.5)$ & \\
\hline $\mathrm{NE}$ & $6(5 \cdot 7)$ & $2(3.0)$ & $4(10.5)$ & \\
\hline R-ISS stage & & & & $0.092^{b}$ \\
\hline I & $3(2.9)$ & $1(1.5)$ & $2(5 \cdot 3)$ & \\
\hline II & $60(57.1)$ & $44(65.7)$ & $16(42.1)$ & \\
\hline III & $39(37.1)$ & $21(31.3)$ & $18(47.4)$ & \\
\hline
\end{tabular}


Table 1. Continued

\begin{tabular}{|c|c|c|c|c|}
\hline \multirow[b]{2}{*}{ Category } & \multirow[b]{2}{*}{ Total $(\mathrm{n}=105)$} & \multicolumn{3}{|c|}{ Treatment group by dose of MP } \\
\hline & & $\begin{array}{l}\text { The reduced-dose group } \\
\qquad(\mathrm{n}=67)\end{array}$ & $\begin{array}{l}\text { The planned-dose group } \\
\qquad(\mathrm{n}=38)\end{array}$ & $p$ value \\
\hline $\mathrm{NE}$ & $3(2.9)$ & $1(1.5)$ & $2(5 \cdot 3)$ & \\
\hline Underlying disease & $89(84.8)$ & $58(86.6)$ & $31(81.6)$ & $0.494^{\mathrm{b}}$ \\
\hline Cardiac problem & $13(12.4)$ & $8(11.9)$ & $5(13.2)$ & $0.626^{b}$ \\
\hline Renal impairment & $17(16.2)$ & $10(14.9)$ & $7(18.4)$ & $0.537^{\mathrm{b}}$ \\
\hline Hypertension & $56(53 \cdot 3)$ & $36(53.7)$ & $20(52.6)$ & $0.627^{b}$ \\
\hline Diabetes mellitus & $21(20.0)$ & $15(22.4)$ & $6(15.8)$ & $0.538^{b}$ \\
\hline Others & $50(47.6)$ & $34(50.7)$ & $16(42.1)$ & $0.570^{b}$ \\
\hline Calculated Ccr, mL/min & $44.2(2.4-97.5)$ & $39 \cdot 3(2.4-95.0)$ & $47.2(12.3-97.5)$ & $0.060^{a}$ \\
\hline$<30$ & $28(26.7)$ & $20(29.9)$ & $8(21.1)$ & $0.505^{\mathrm{b}}$ \\
\hline $30 \leq \mathrm{Ccr}<60$ & $47(44.7)$ & $30(44.8)$ & $17(44.7)$ & \\
\hline$\geq 60$ & $30(28.6)$ & $17(25 \cdot 4)$ & $13(34.2)$ & \\
\hline Cumulative bortezomib dose, $\mathrm{mg} / \mathrm{m}^{2}$ & $41.6(2.6-67.6)$ & $41.6(2.6-67.6)$ & $40.4(6.5-67.6)$ & $0.925^{\mathrm{a}}$ \\
\hline$<41.6$ & $48(45 \cdot 7)$ & $29(43 \cdot 3)$ & $19(50.0)$ & $0.507^{b}$ \\
\hline$\geq 41.6$ & $57(54 \cdot 3)$ & $38(56.7)$ & $19(50.0)$ & \\
\hline Cumulative melphalan dose & $108(18-324)$ & $99(18-252)$ & $144(36-324)$ & $0.020^{\mathrm{a}}$ \\
\hline$<50 \%$ & $67(63.8)$ & $47(70.1)$ & $20(52.6)$ & $<0.001^{\mathrm{b}}$ \\
\hline $50 \% \leq \mathrm{M}<75 \%$ & $13(12.4)$ & $11(16.4)$ & $2(5 \cdot 3)$ & \\
\hline $75 \% \leq M<100 \%$ & $14(13 \cdot 3)$ & $9(13.4)$ & $5(13.2)$ & \\
\hline $100 \%$ of planned dose & $11(10.5)$ & o & $11(28.9)$ & \\
\hline Cumulative prednisone dose & $900(120-2,160)$ & $720(120-2,160)$ & $960(240-2,160)$ & $0.066^{\mathrm{a}}$ \\
\hline$<50 \%$ & $62(60.0)$ & $42(62.7)$ & $20(52.6)$ & $0.001^{b}$ \\
\hline $50 \% \leq \mathrm{PRD}<75 \%$ & $11(10.5)$ & $10(14.9)$ & $1(2.6)$ & \\
\hline $75 \% \leq \mathrm{PRD}<100 \%$ & $15(14 \cdot 3)$ & $11(16.4)$ & $4(10.5)$ & \\
\hline $100 \%$ of planned dose & $17(15.2)$ & $4(6.0)$ & $13(34.2)$ & \\
\hline Weekly bortezomib, initially & $7(6.7)$ & $4(6.0)$ & $3(7.9)$ & $0.704^{b}$ \\
\hline Changes to weekly schedule & $46(43.8)$ & $28(41.8)$ & $18(47 \cdot 4)$ & $0.580^{b}$ \\
\hline
\end{tabular}

Values are presented as median (range) or number (\%).

MP, melphalan-prednisone; BSA, body surface area; ECOG PS, Eastern Cooperative Oncology Group performance status; Ig, immunoglobulin; $\beta 2-\mathrm{MG}, \beta 2$-microglobulin; NE, not evaluated; ISS, International Staging System; R-ISS, revised-International

Staging System; Ccr, creatinine clearance; M, melphalan; PRD, prednisone.

${ }^{a}$ Mann-Whitney U test.

${ }^{\mathrm{b}}$ Chi-square test.

${ }^{\mathrm{c}}$ Risk group by fluorescence in situ hybridization and conventional cytogenetics.

and other reasons for the remaining five patients. The median number of cycles delivered was four (range, 1 to 9 ), and the median cumulative dose of bortezomib was $41.6 \mathrm{mg} / \mathrm{m}^{2}$ (range, 2.6 to 67.6 ). The median cumulative doses of melphalan and prednisone were $108 \mathrm{mg} / \mathrm{m}^{2}$ (range, 18 to 324 ) and $900 \mathrm{mg} / \mathrm{m}^{2}$ (range, 120 to 2,160 ), respectively.

Differences in baseline characteristics between the reduced- and planned-dose groups are shown in Table 1. There was no significant difference in the cumulative dose of bortezomib between the two groups (41.6 $\mathrm{mg} / \mathrm{m}^{2}$ vs. $40.4 \mathrm{mg} / \mathrm{m}^{2}$ in the reduced- and planned-dose 
Table 2. Best response to bortezomib plus melphalan-prednisone treatment

\begin{tabular}{|c|c|c|c|c|}
\hline \multirow[b]{2}{*}{ Category $^{\mathrm{a}}$} & \multirow{2}{*}{$\begin{array}{l}\text { Total patients } \\
\qquad(\mathrm{n}=105)\end{array}$} & \multicolumn{2}{|c|}{ Treatment group by dose of MP } & \multirow[b]{2}{*}{$p$ value } \\
\hline & & $\begin{array}{l}\text { The reduced-dose group } \\
\qquad(\mathrm{n}=67)\end{array}$ & $\begin{array}{l}\text { The planned-dose group } \\
\qquad(\mathrm{n}=38)\end{array}$ & \\
\hline Stringent CR & $20(19.0)$ & $14(20.9)$ & $6(15.8)$ & $0.711^{b}$ \\
\hline $\mathrm{CR}$ & $12(11.4)$ & $7(10.4)$ & $5(13.2)$ & \\
\hline VGPR & $10(9.5)$ & $7(10.4)$ & $3(7 \cdot 9)$ & \\
\hline $\mathrm{PR}$ & $43(41.0)$ & $27(40.3)$ & $16(42.1)$ & \\
\hline $\mathrm{MD}$ & $11(10.5)$ & $6(9.0)$ & $5(13.2)$ & \\
\hline $\mathrm{SD}$ & $3(2.9)$ & $3(4 \cdot 5)$ & o & \\
\hline $\mathrm{PD}$ & $1(1.0)$ & o & $1(2.6)$ & \\
\hline $\mathrm{NE}$ & $5(4.8)$ & $3(4 \cdot 5)$ & $2(5 \cdot 3)$ & \\
\hline $\mathrm{ORR}(\geq \mathrm{PR})$ & $85(81.0)$ & $55(82.0)$ & $30(79.0)$ & \\
\hline $\mathrm{CRR}(\mathrm{sCR}+\mathrm{CR})$ & $32(30.4)$ & $21(31.3)$ & $11(29.0)$ & \\
\hline
\end{tabular}

Values are presented as number (\%).

MP, melphalan-prednisone; CR, complete response; VGPR, very good partial response; PR, partial response; MD, minimal response; $\mathrm{SD}$, stable disease; $\mathrm{PD}$, progressive disease; $\mathrm{NE}$, not evaluated; ORR, overall response rate; CRR, complete response rate; $\mathrm{sCR}$, stringent complete response.

${ }^{a}$ Category of response on the basis of International Uniform Response Criteria.

${ }^{\mathrm{b}}$ Chi-square test.

groups, respectively; $p=0.925)$. In contrast, the median cumulative dose of melphalan in the reduced-dose group was significantly lower than that in the planneddose group (99 mg/m² vs. $144 \mathrm{mg} / \mathrm{m}^{2}$, respectively; $p=$ o.020). Slightly higher cumulative doses of prednisone were administrated in the planned-dose group (960 $\mathrm{mg} / \mathrm{m}^{2}$ vs. $720 \mathrm{mg} / \mathrm{m}^{2}$, respectively; $p=0.060$ ). According to the median dose intensity by the number of cycle, the median dose intensity for bortezomib was $8.1 \mathrm{mg} /$ $\mathrm{m}^{2} /$ cycle (range, 2.6 to 10.4) and did not differ between the reduced- and the planned-dose groups $\left(8.2 \mathrm{mg} / \mathrm{m}^{2}\right)$ cycle vs. $7.7 \mathrm{mg} / \mathrm{m}^{2} /$ cycle, respectively; $\left.p=0.373\right)$. Unlike bortezomib, the median dose intensities for melphalan and prednisone were lower in the reduced-dose group than those in the planned-dose group (melphalan: 24.5 $\mathrm{mg} / \mathrm{m}^{2} /$ cycle vs. $35.4 \mathrm{mg} / \mathrm{m}^{2} /$ cycle, respectively, $\mathrm{p}<0.001$; prednisone: $191.0 \mathrm{mg} / \mathrm{m}^{2} /$ cycle vs. $236.7 \mathrm{mg} / \mathrm{m}^{2} /$ cycle, respectively, $p<0.001$ ). In this study, approximately $50 \%$ of patients were treated on a weekly schedule. In this regard, the median dose intensity of bortezomib was similar to that of the planned dose intensity of the VISTA trial ( $1.23 \mathrm{mg} / \mathrm{m}^{2} /$ week vs. $1.25 \mathrm{mg} / \mathrm{m}^{2} /$ week, respectively) and there was no difference between the two dose groups $(p=0.853)$. In contrast, the median dose intensi- ties of melphalan and prednisone were lower than those of the VISTA trial (melphalan: $4.1 \mathrm{mg} / \mathrm{m}^{2} /$ week vs. 6.0 $\mathrm{mg} / \mathrm{m}^{2} /$ week, respectively; prednisone: $30.7 \mathrm{mg} / \mathrm{m}^{2} /$ week vs. $40.0 \mathrm{mg} / \mathrm{m}^{2} /$ week, respectively). The median dose intensity of MP in the reduced-dose group was lower than that in the planned-dose group (melphalan: $3.6 \mathrm{mg} / \mathrm{m}^{2}$ / week vs. $5.5 \mathrm{mg} / \mathrm{m}^{2} /$ week, respectively, $p<0.001$; prednisone: $29.0 \mathrm{mg} / \mathrm{m}^{2} /$ week vs. $37.2 \mathrm{mg} / \mathrm{m}^{2} /$ week, respective$l y, p=0.001$ ). With the exception of the difference in administered MP dose, there was no significant difference in baseline characteristics between the dose groups.

\section{Treatment response}

The overall response rate (ORR) and complete response rate (CRR) (i.e., stringent complete response [CR] and CR) were observed to be $81.0 \%$ and $30.4 \%$, respectively. Details regarding the responses to treatments are shown in Table 2. Patients receiving less than 50\% of the planned MP dose showed a lower incidence of CR compared with patients receiving $\geq 50 \%$ of the planned MP dose (melphalan: $31.2 \%$ vs. 68.8\%, respectively, $p<0.001$; prednisone: $25.0 \%$ vs. $75.0 \%$, respectively, $p<0.001$ ). Although more patients in the reduced-dose group received less than $50 \%$ of the planned MP dose, the ORR and CRR 
Table 3. Adverse events of all patients, and adverse events for patients in groups with different MP doses

\begin{tabular}{|c|c|c|c|c|}
\hline \multirow[b]{2}{*}{ Category } & \multirow[b]{2}{*}{ Total $(n=105)$} & \multicolumn{3}{|c|}{ Treatment group by dose of MP } \\
\hline & & $\begin{array}{l}\text { The reduced-dose group } \\
\qquad(\mathrm{n}=67)\end{array}$ & $\begin{array}{l}\text { The planned-dose group } \\
\qquad(\mathrm{n}=38)\end{array}$ & $p$ value \\
\hline$\geq$ Grade $3-4$ toxicity & $75(71.4)$ & $50(74.6)$ & $25(65.8)$ & $0.335^{\mathrm{a}}$ \\
\hline \multicolumn{5}{|l|}{ Time to occurrence, day } \\
\hline Hematologic toxicity & $27(3-322)$ & $31(3-322)$ & $22(5-273)$ & $0.067^{\mathrm{b}}$ \\
\hline Non-hematologic toxicity & $57(7-1,169)$ & $63(7-1,169)$ & $56(8-436)$ & $0.293^{\mathrm{b}}$ \\
\hline$\geq$ Grade $3-4$ hematologic toxicity & $68(64.8)$ & $45(67.2)$ & $23(60.5)$ & $0.494^{\mathrm{a}}$ \\
\hline Hemoglobin & $22(21.0)$ & $16(23.9)$ & $6(15.8)$ & $0.728^{\mathrm{a}}$ \\
\hline Neutrophil & $43(40.9)$ & $24(35.8)$ & $19(50.0)$ & $0.451^{\mathrm{a}}$ \\
\hline Platelet & $25(23.9)$ & $15(22.4)$ & $10(26.4)$ & $0.164^{a}$ \\
\hline$\geq$ Grade 3-4 non-hematologic toxicity & $27(25.7)$ & $19(28.4)$ & $8(21.1)$ & $0.410^{\mathrm{a}}$ \\
\hline Peripheral neuropathy, any grade & $11(10.5)$ & $7(10.4)$ & $4(10.5)$ & $0.616^{c}$ \\
\hline$\geq$ Grade $3-4$ & $5(4.8)$ & $3(4.5)$ & $2(5.3)$ & $0.819^{\mathrm{a}}$ \\
\hline Diarrhea, any grade & $5(4.8)$ & $4(3.8)$ & $1(1.0)$ & $0.651^{c}$ \\
\hline$\geq$ Grade $3-4$ & $2(1.9)$ & $1(1.5)$ & $1(2.6)$ & $0.171^{\mathrm{a}}$ \\
\hline Infection, any grade & $31(29.5)$ & $22(32.8)$ & $9(23.7)$ & $0.323^{\mathrm{a}}$ \\
\hline$\geq$ Grade $3-4$ & $17(16.1)$ & $12(17.9)$ & $5(13.1)$ & $0.959^{\mathrm{a}}$ \\
\hline Pneumonia, any grade & $16(15.2)$ & $10(14.9)$ & $6(15.8)$ & $0.906^{\mathrm{a}}$ \\
\hline$\geq$ Grade $3-4$ & $11(10.5)$ & $7(10.4)$ & $4(10.5)$ & $0.889^{\mathrm{a}}$ \\
\hline Herpes zoster, any grade & $7(6.7)$ & $5(7.5)$ & $2(5 \cdot 3)$ & $0.503^{c}$ \\
\hline$\geq$ Grade $3-4$ & $1(1.0)$ & $1(1.5)$ & o & $0.495^{\mathrm{a}}$ \\
\hline Other infections, any grade & $9(8.6)$ & $7(10.4)$ & $2(5.3)$ & $0.483^{c}$ \\
\hline$\geq$ Grade $3-4$ & $5(4.8)$ & $3(4.5)$ & $2(5 \cdot 3)$ & $0.151^{\mathrm{a}}$ \\
\hline \multicolumn{5}{|l|}{ Dose change of VMP } \\
\hline Due to hematologic toxicity & $10(9.5)$ & $4(6.0)$ & $6(15.8)$ & $0.163^{c}$ \\
\hline Due to non-hematologic toxicity & $19(18.1)$ & $11(16.4)$ & $8(21.1)$ & $0.553^{\mathrm{a}}$ \\
\hline Changes to weekly schedule & $46(43.8)$ & $28(41.8)$ & $18(47.4)$ & $0.580^{\mathrm{a}}$ \\
\hline \multicolumn{5}{|l|}{ Discontinuation of VMP } \\
\hline Due to hematologic toxicity & $1(1.0)$ & $1(1.5)$ & o & $0.646^{c}$ \\
\hline Due to non-hematologic toxicity & $25(26.0)$ & $17(27.4)$ & $8(23.5)$ & $0.678^{a}$ \\
\hline
\end{tabular}

Values are presented as number (\%) or median (range).

MP, melphalan-prednisone; VMP, bortezomib plus melphalan-prednisone.

${ }^{\mathrm{a}}$ Chi-square test.

${ }^{\mathrm{b}}$ Mann-Whitney U test.

${ }^{\mathrm{c}}$ Fisher's exact test.

were not significantly different between the reducedand planned-dose groups $(p=0.711)$. This could be explained by the fact that the median cumulative dose and dose intensity of bortezomib did not differ between the two groups. The median DOR after a partial response had been achieved was 17.8 months for the overall pop- ulation. There was no statistically significant difference in DOR between the two dose groups $(p=0.712)$. However, the median DOR of patients who achieved CR was longer than that of patients who did not show CR (42.2 months vs. 12.2 months, respectively; $p<0.001$ ). A total of 25 patients (37.3\%) in the reduced-dose group and 16 
patients (42.1\%) in the planned-dose group received subsequent therapy following $\operatorname{VMP}(p=0.549)$. The time to next treatment was not significantly different between the reduced- and planned-dose groups (10.2 months vs. 12.8 months, respectively; $p=0.935)$. Lenalidomide was the most common agent used in subsequent therapy in both reduced- and planned-dose group patients $(22.4 \%$ vs. $26.3 \%$ of patients, respectively), followed by thalidomide (9.0\% vs. $10.5 \%$, respectively), bortezomib (4.5\% vs. $5.3 \%$, respectively), and others including carfilzomib (3.0\% vs. ०\%, respectively).

\section{Adverse events}

The overall incidence of grade 3 to 4 or greater adverse events among our 105 patients was $71.4 \%$. The proportion of hematologic toxicities of grade 3 to 4 or greater was higher than that of non-hematologic toxicities $(64.8 \%$ vs. $25.7 \%$, respectively). However, changes in VMP dose due to non-hematologic toxicity were more common (18.1\% vs. 9.5\%, respectively). The treatment-emergent adverse events reported for patients in the reduced- and planned-dose groups are summarized in Table 3. The overall incidence of adverse events, time to occurrence, and the proportions of dose change and discontinuation of VMP due to adverse events were similar in both dose groups.

The main reason for a further reduction in VMP doses in this study was occurrence of adverse events. In the reduced-dose group, 14 patients (20.9\%) had additional reduction of the VMP dose. The $1.3 \mathrm{mg} / \mathrm{m}^{2}$ dose of bortezomib was reduced to a dose of $1.0 \mathrm{mg} / \mathrm{m}^{2}$ in six patients and the MP dose was further reduced by $25 \%$ from the initial dose in six patients. The remaining two patients had both modified doses of bortezomib and MP. Furthermore, 13 patients (34.2\%) in the planneddose group received an additional dose reduction. Five of the 13 patients were treated with bortezomib (at a dose of $1.0 \mathrm{mg} / \mathrm{m}^{2}$ ) and six received a modified dose of MP in accordance with the above reduction method. The remaining two patients in this dosing group received modified doses of both bortezomib and MP. The main reasons for changes in bortezomib dose were adverse events in 18 patients, which included five general weakness, four thrombocytopenia, two neutropenia, two peripheral neuropathy, two herpes zoster, and three oth- ers. The dose of MP was further reduced in 16 patients, which included three with general weakness, three with neutropenia, two with thrombocytopenia, two with decreased renal function, two with pneumonia, and four others. During the treatment, 53 patients (50.5\%), including those receiving initial weekly bortezomib had maintained the weekly schedule of bortezomib. The main reasons for these changes to weekly schedules were also adverse events, which included general weakness in 16 patients $(34.8 \%)$, peripheral neuropathy in 13 patients (28.3\%), diarrhea in six patients $(13.0 \%)$, thrombocytopenia and neutropenia in four patients (8.7\%), patient's choice in two patients (4.3\%), and other reasons for the remaining nine patients (17.3\%).

Due to adverse events, 26 patients $(27.1 \%)$ discontinued treatment. Among these, the proportion who discontinued treatment due to non-hematologic toxicities was higher than that due to hematologic toxicities $(26.0 \%$ vs. $1.0 \%$, respectively). These findings suggest that the hematologic toxicities were clinically manageable in most patients. Although there was no difference between dose groups in terms of the percentage of patients who discontinued treatment due to adverse events $(p=0.452)$, patients in the planned-dose group experienced more discontinuation due to adverse events within two cycles than in the reduced-dose group (80\% vs. $38.5 \%, p=0.016$ ).

\section{Survival data}

The median duration of follow-up for the 105 patients was 15.7 months. The median PFS of all patients was 25.0 months (95\% confidence interval [CI], 7.3 to 42.7 ) (Fig. 1A). The median PFS of the reduced-dose group was 36.0 months, which was longer than that of the planned-dose group (20.2 months), although the difference was not statistically significant $(p=0.335)$ (Fig. $1 \mathrm{~B})$. The median OS of all patients was 47.8 months (95\% CI, 26.5 to 69.1) (Fig. $2 \mathrm{~A})$. Although the median OS in the reduced-dose group was longer than that in the planned-dose group, this difference was not statistically significant (53.1 months vs. 40.4 months, $p=0.576$ ) (Fig. 2B). During the follow-up period, 35 patients (33.3\%) died, although the death rate did not differ significantly between the two dose groups $(p=0.566)$. The median PFS was significantly longer in patients in the higher $\left(\geq 41.6 \mathrm{mg} / \mathrm{m}^{2}\right)$ versus the lower $\left(<41.6 \mathrm{mg} / \mathrm{m}^{2}\right)$ cumulative bortezomib dose group (35.1 months vs. 9.6 months, $p<0.001)$. Although there was 

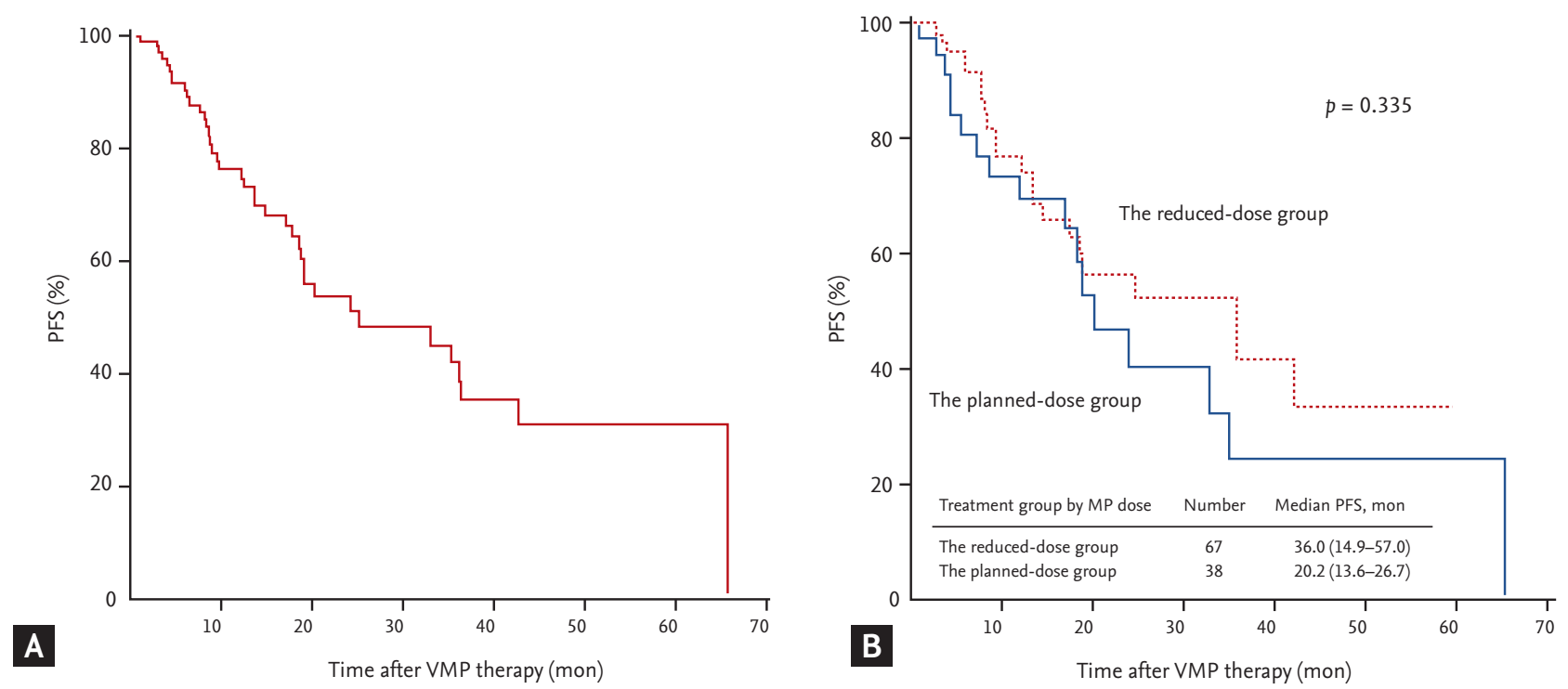

Figure 1. The progression-free survival (PFS) of all patients (A) and PFS of patients divided based on the dose of melphalan-prednisone (MP) they received (B). VMP, bortezomib plus melphalan-prednisone.
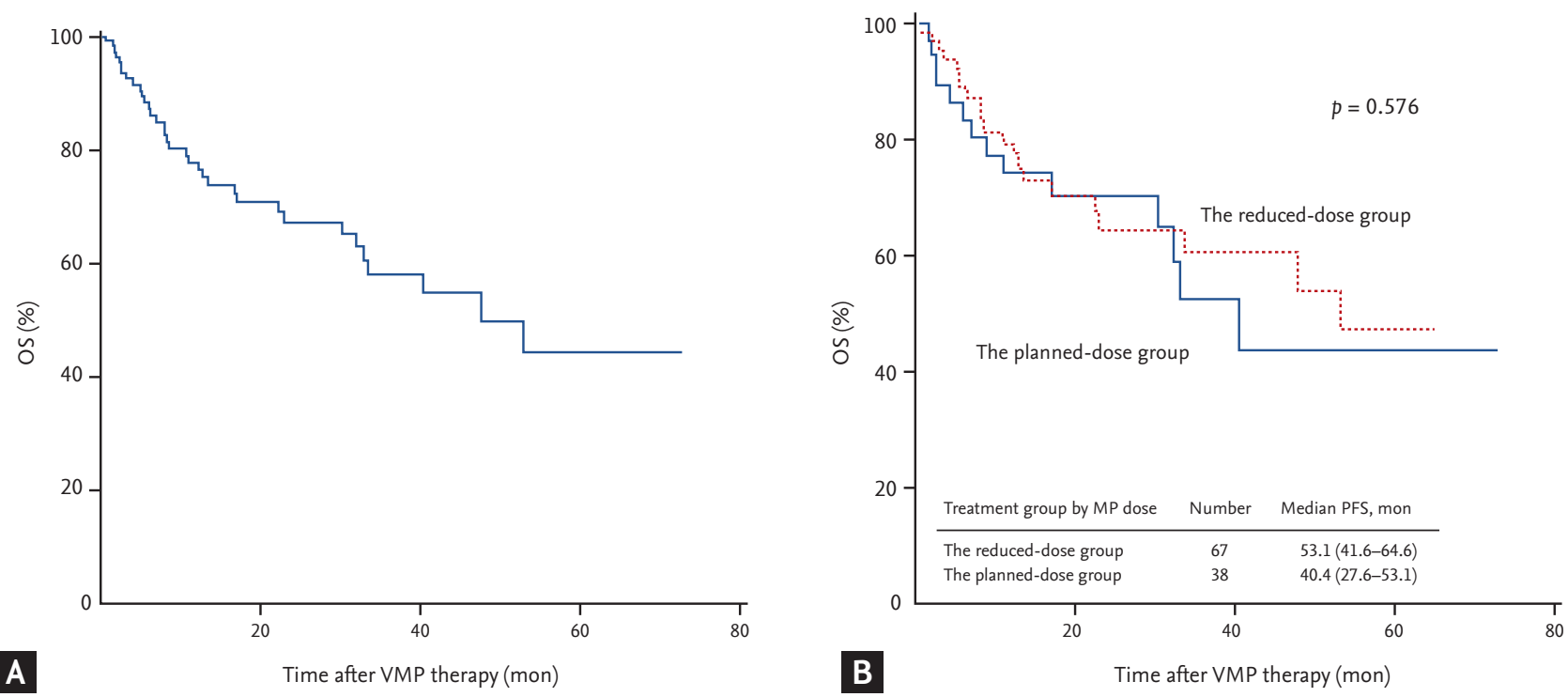

Figure 2. The overall survival (OS) of all patients (A) and OS of patients divided based on the dose of melphalan-prednisone (MP) they received (B). VMP, bortezomib plus melphalan-prednisone.

no difference in PFS due to cumulative melphalan dose, there was a difference in PFS related to median cumulative prednisone dose. The median PFS in the higher $\left(\geq 900 \mathrm{mg} / \mathrm{m}^{2}\right.$ ) prednisone dose group was significantly longer than that in the lower $\left(<900 \mathrm{mg} / \mathrm{m}^{2}\right)$ prednisone dose group (35.1 months vs. 13.5 months, respectively; $p=$ 0.005). Univariate analyses, performed to assess whether the amount of MP dose affected PFS, revealed that patients receiving less than $50 \%$ of the planned MP dose had a significantly shorter PFS compared with those receiving $50 \%$ to $99 \%$ of the planned dose (melphalan: 13.5 months vs. not reached, $p<0.001$; prednisone: 12.3 months vs. not reached, $p=0.002$, respectively) (Fig. 3). The median PFS of the patients treated for more than four cycles was significantly longer than that of patients treated with fewer than four cycles (33.0 months vs. 13.5 months, respectively; $p=0.002$ ). Multivariate analysis showed that the cumulative dose of bortezomib $<41.6$ 

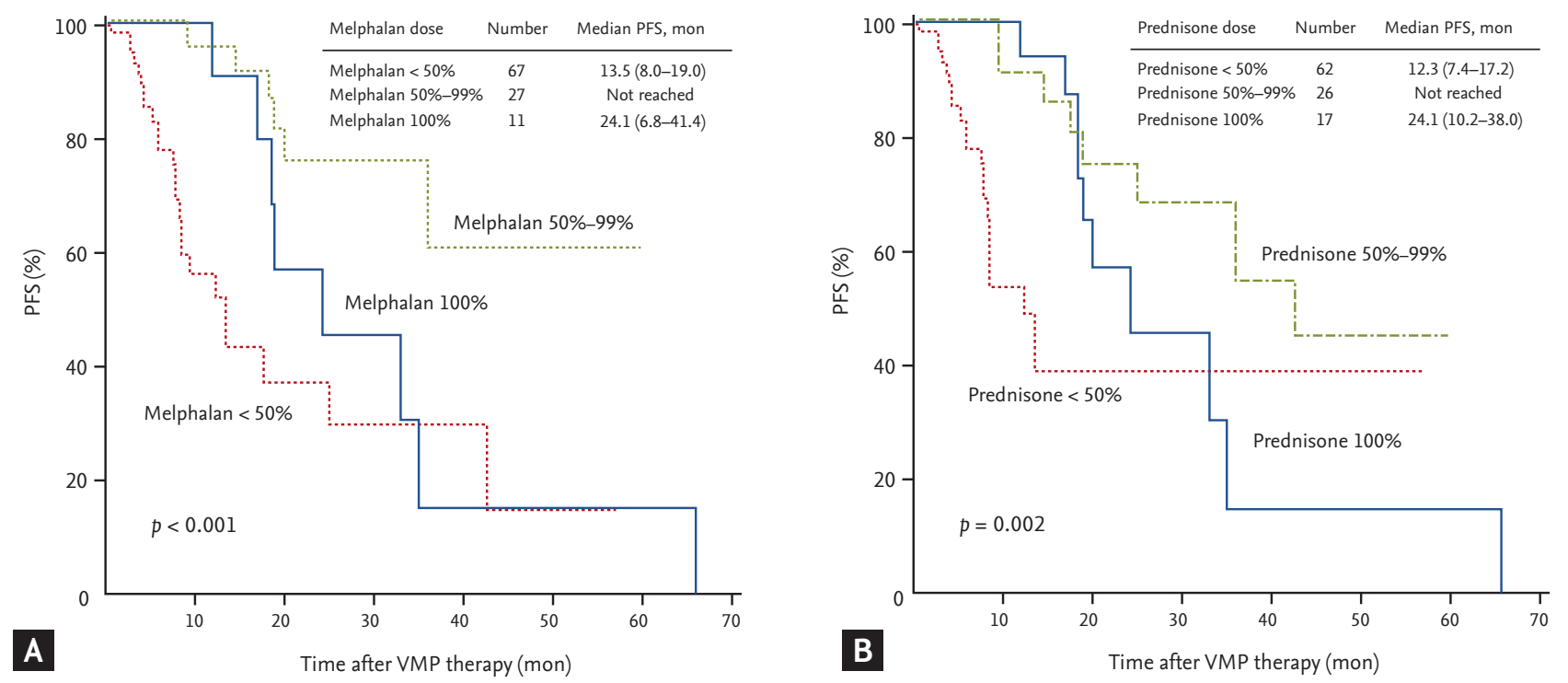

Figure 3. The progression-free survival (PFS) of all patients. Patients divided based on the planned dose of melphalan (A). Patients divided based on the planned dose of prednisone (B). VMP, bortezomib plus melphalan-prednisone.

Table 4. Prognostic factors for progression-free survival

\begin{tabular}{|c|c|c|c|c|c|}
\hline \multirow{2}{*}{ Factor } & \multicolumn{2}{|c|}{ Univariate analysis } & \multicolumn{3}{|c|}{ Multivariate analysis } \\
\hline & PFS, mon & $p$ value $^{a}$ & HR & $95 \% \mathrm{CI}$ & $p$ value $^{\mathrm{b}}$ \\
\hline Median cycles of VMP & & 0.002 & & & \\
\hline$<4$ cycle & 13.5 & & 1 & & \\
\hline$\geq 4$ cycle & 33.0 & & 0.574 & $0.122-2.707$ & 0.483 \\
\hline Cumulative bortezomib dose, $\mathrm{mg} / \mathrm{m}^{2}$ & & $<0.001$ & & & \\
\hline$<41.6$ & 9.6 & & 3.881 & $1.092-13.790$ & 0.036 \\
\hline$\geq 41.6$ & 35.1 & & 1 & & \\
\hline Cumulative melphalan dose, $\mathrm{mg} / \mathrm{m}^{2}$ & & 0.169 & & & \\
\hline$<108$ & 13.6 & & & & \\
\hline$\geq 108$ & 33.0 & & & & \\
\hline Cumulative prednisone dose, $\mathrm{mg} / \mathrm{m}^{2}$ & & 0.005 & & & \\
\hline$<900$ & $13 \cdot 5$ & & 1.749 & $0.657-4.656$ & 0.263 \\
\hline$\geq 900$ & 35.1 & & 1 & & \\
\hline
\end{tabular}

PFS, progression-free survival; HR, hazard ratio; CI, confidence interval; VMP, bortezomib plus melphalan-prednisone.

${ }^{\mathrm{a}}$ Log-rank test.

${ }^{\mathrm{b}}$ Cox proportional hazard model.

$\mathrm{mg} / \mathrm{m}^{2}$ (vs. $\geq 41.6 \mathrm{mg} / \mathrm{m}^{2}$ ) was the only significant independent predictor of shorter PFS (hazard ratio, 3.881; 95\% CI, 1.1 to $13.8 ; p=0.036$ ) (Table 4 ).

Univariate analyses of the factors affecting OS, revealed that ECOG PS, underlying cardiac disease, calculated creatinine clearance, median VMP cycle, median cumulative dose of VMP, and the occurrence of grade 3 to 4 non-hematologic toxicities were all significantly associated with OS. The median OS in the group with the higher $\left(\geq 41.6 \mathrm{mg} / \mathrm{m}^{2}\right)$ cumulative bortezomib dose was not reached (vs. 11.0 months in the lower dose group, $p$ $<0.001)$. Univariate analysis, performed to assess wheth- 

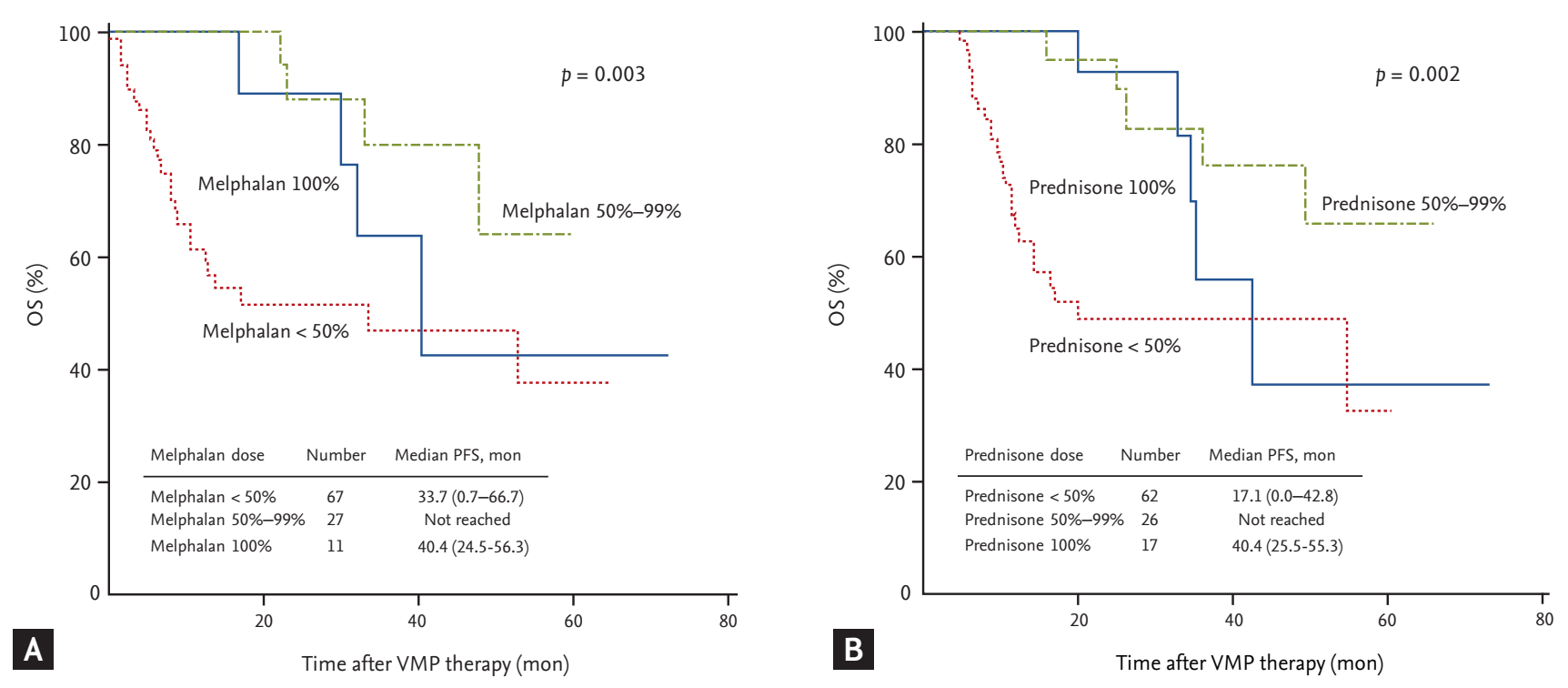

Figure 4. The overall survival (OS) of all patients. Patients divided based on the planned dose of melphalan (A). Patients divided based on the planned dose of prednisone (B). VMP, bortezomib plus melphalan-prednisone.

er the amount of the MP dose affected OS, showed that patients receiving less than $50 \%$ of the planned MP dose had a significantly shorter OS compared with those receiving $50 \%$ to $99 \%$ of the planned dose (melphalan: 33.7 months vs. not reached, $p=0.003$; prednisone: 17.1 months vs. not reached, $p=0.002$, respectively) (Fig. 4). Multivariate analysis of the factors affecting OS revealed that underlying cardiac disease, median VMP cycle $(<4$ cycles), and occurrence of grade 3 to 4 non-hematologic toxicity were all significant independent predictors of shorter OS (Table 5).

\section{DISCUSSION}

One means of achieving more satisfactory outcomes in elderly MM patients is to better define the dose reductions required when using standard treatment regimens. From the onset of VMP therapy, if the VMP dose is appropriately reduced, the therapy can be continued without adverse effects. This will increase the level of response to treatment and prolong patient survival. In our study, most patients received MP doses that were less than the maximum planned dose. Only approximately $30 \%$ of the patients received the maximum planned dose of MP, even in the planned-dose group. In contrast, the median cumulative dose of bortezomib $\left(41.6 \mathrm{mg} / \mathrm{m}^{2}\right)$ was slightly higher than the $39 \mathrm{mg} / \mathrm{m}^{2}$ value used in the posthoc landmark analysis of the VISTA study [6].

Safety profiles were consistent with the known toxicities of bortezomib and MP reported previously [4,12]. Compared with the VISTA trial, which included only approximately $10 \%$ of Asian patients [4], the incidence of similar grade 3 to 4 or greater hematologic toxicities in our study was surprising, and even lower than that of thrombocytopenia (19.0\% vs. $21.0 \%$ for anemia, $40.0 \%$ vs. $40.9 \%$ for neutropenia, and $37.0 \%$ vs. $23.9 \%$ for thrombocytopenia in VISTA and our study, respectively). These frequencies are considerably lower than those in a phase I/II study of Japanese patients in the same Asian population (35\% for anemia, $67 \%$ for neutropenia, and $49 \%$ for thrombocytopenia) [13]. The largest component of grade 3 to 4 or greater non-hematologic toxicities was infection (15.3\%), and pneumonia was the most common cause of the discontinuation of treatment. Incidences of peripheral neuropathy and herpes zoster were found to be relatively lower than those reported in previous studies $[4,7,13,14]$. The relatively lower incidence of peripheral neuropathy in this study might have been due to the change to subcutaneous bortezomib administration from an early period in the study and also weekly dosing. The lower incidence of herpes zoster observed might be explained by the fact that all patients received prophylactic acyclovir treatment. The proportion of dis- 
Table 5. Prognostic factors for overall survival

\begin{tabular}{|c|c|c|c|c|c|}
\hline \multirow{2}{*}{ Factor } & \multicolumn{2}{|c|}{ Univariate analysis } & \multicolumn{3}{|c|}{ Multivariate analysis } \\
\hline & OS, mon & $p$ value $^{a}$ & HR & $95 \%$ CI & $p$ value $^{\mathrm{b}}$ \\
\hline ECOG PS & & 0.001 & & & \\
\hline $0-1$ & 53.1 & & 1 & $0.586-3.975$ & 0.387 \\
\hline$\geq 2$ & 12.8 & & 1.526 & & \\
\hline Underlying cardiac disease & & 0.019 & & & \\
\hline Yes & 8.8 & & 3.032 & $1.182-7.777$ & 0.021 \\
\hline No & 53.1 & & 1 & & \\
\hline Calculated Ccr, mL/min & & 0.033 & & & 0.088 \\
\hline$<30$ & 17.1 & & 2.337 & $0.876-6.233$ & 0.090 \\
\hline $30-60$ & Not reached & & 0.987 & $0.376-2.591$ & 0.979 \\
\hline$\geq 60$ & Not reached & & 1 & & \\
\hline Median cycles of VMP & & $<0.001$ & & & \\
\hline$<4$ cycle & 8.1 & & 23.543 & $1.394-39.493$ & 0.028 \\
\hline$\geq 4$ cycle & Not reached & & 1 & & \\
\hline Cumulative bortezomib dose, $\mathrm{mg} / \mathrm{m}^{2}$ & & $<0.001$ & & & \\
\hline$<41.6$ & 11.0 & & 1 & & \\
\hline$\geq 41.6$ & Not reached & & 0.409 & $0.037-4.531$ & 0.466 \\
\hline Cumulative melphalan dose, $\mathrm{mg} / \mathrm{m}^{2}$ & & $<0.001$ & & $0.243-3.006$ & 0.806 \\
\hline$<108$ & 11.0 & & 0.854 & & \\
\hline$\geq 108$ & Not reached & & 1 & & \\
\hline Cumulative prednisone dose, $\mathrm{mg} / \mathrm{m}^{2}$ & & $<0.001$ & & & \\
\hline$<900$ & 13.6 & & 0.628 & $0.119-3.298$ & 0.582 \\
\hline$\geq 900$ & Not reached & & 1 & & \\
\hline Grade 3-4 non-hematologic toxicity & & $<0.001$ & & & \\
\hline Yes & 8.4 & & 5.125 & $2.132-12.319$ & $<0.001$ \\
\hline No & 53.1 & & 1 & & \\
\hline
\end{tabular}

OS, overall survival; HR, hazard ratio; CI, confidence interval; ECOG PS, Eastern Cooperative Oncology Group performance status; Ccr, creatinine clearance; VMP, bortezomib plus melphalan-prednisone.

${ }^{\mathrm{a}}$ Log-rank test.

${ }^{\mathrm{b}}$ Cox proportional hazard model.

continued treatment due to adverse events was relatively lower than that recorded in previous studies $[5,13,14]$, and in this regard, the only difference between the dose groups was that more patients discontinued within two cycles due to adverse events in the planned-dose group. These results indicate that it may be possible to continue treatment with a modified dose of MP.

The ORR and CRR were similar in both dose groups. The recorded CRR of $30.5 \%$ is comparable that of approximately $30 \%$ observed in previous studies [4,14]. Moreover, our CRR was higher than that obtained in an- other Korean study with similar baseline characteristics and a Japanese study (22\% and 19\%, respectively), and our ORR of $81.0 \%$ was also higher than that obtained in these two studies $[7,13]$. In addition to the cumulative dose, the median dose intensity for bortezomib in this study $\left(8.1 \mathrm{mg} / \mathrm{m}^{2} /\right.$ cycle) is comparable to that of the VISTA study $\left(8.32 \mathrm{mg} / \mathrm{m}^{2} /\right.$ cycle $)$ and higher than that of the aforementioned Japanese study $\left(6.86 \mathrm{mg} / \mathrm{m}^{2} /\right.$ cycle $)$ [13]. The median number of cycles in this study was four (range, 1 to 9 cycles), which is similar to that in the previously mentioned Japanese study [13], and also to that in 
another Korean observational study ( 4.5 and 5 cycles, respectively) [7]. Our median four cycles is one-half of the median number of cycles used in the VISTA trial, but did not differ between the reduced- and the planneddose groups. Although the median number of cycles was lower, the median PFS of all patients was 25.0 months, which is similar to the 24 months PFS of the VISTA trial [4]. These results indicate that the cumulative dose and dose intensity of bortezomib administered is important for treatment response and survival outcomes, which can be achieved through treatment modification, including a modified dose of MP and weekly schedule of bortezomib.

A recent analysis of VMP data from VISTA and a prospective multicenter Korean observational study indicate that a higher cumulative bortezomib dose is associated with superior treatment outcomes $[6,7]$. In our study, the median PFS and OS were also significantly longer in patients receiving a higher $\left(\geq 41.6 \mathrm{mg} / \mathrm{m}^{2}\right) \mathrm{cu}$ mulative bortezomib dose. In our multivariate analysis of PFS, a cumulative dose of bortezomib $<41.6 \mathrm{mg} / \mathrm{m}^{2}$ was the only significant independent predictor of shorter PFS. In contrast to the clinical results for bortezomib dose, clinical outcomes according to MP dose were different. This difference in PFS could be explained by the lower incidence of CR in patients receiving less than $50 \%$ of the planned MP dose. Additionally, patients receiving less than $50 \%$ of the planned MP dose had a shorter OS compared with those receiving 50\% to $99 \%$ of the planned dose. These results could be explained by the higher incidence of non-hematologic toxicities, including pneumonia, which is the most common cause of the discontinuation of VMP, in the lower dose groups. Patients with myeloma are at an increased risk of infection [15], and the rate of infection is highest in the first 3 to 4 months of therapy induction and in the setting of relapsed disease $[15,16]$. If the dose of MP is set too low, this would not lead to an appropriate therapeutic response; the clinical symptoms of MM would consequently not be regulated, and the chances of infection might also increase. Therefore, these results indicate that even if a reduced-dose of MP is used initially, the MP dose should not be reduced to less than $50 \%$ of the planned dose in order to achieve treatment-related improvements in the disease and prolonged survival outcomes.
In conclusion, administering a modified dose of MP in VMP might be a feasible and effective approach for the treatment of Korean patients with newly diagnosed myeloma who are ineligible for high-dose therapy. Modification of the MP dose appeared to contribute to the avoidance of early discontinuation due to adverse events. It could also enable a continuation of treatment, which can promote higher CRR and prolonged survival through receipt of an effective dose of bortezomib. However, attention should be paid to ensuring that the MP dose does not reduce to less than $50 \%$ of the planned dose, so as to balance risks and benefits of this strategy. In the future, a well-designed prospective study will be required to confirm whether this approach is appropriate in the examined MM population.

\section{KEY MESSAGE}

1. Modifying the dose of melphalan-prednisone might be a feasible and effective approach for Korean patients receiving bortezomib plus melphalan-prednisone treatment.

2. Through modifying the dose of melphalan-prednisone, we will be able to avoid early treatment discontinuation and continue treatment without adversely affecting the clinical outcomes.

3. Nevertheless, careful attention must be paid to ensuring that the modified dose of melphalan-prednisone is not less than $50 \%$ of the planned dose.

\section{Conflict of interest}

No potential conflict of interest relevant to this article was reported.

\section{REFERENCES}

1. Kyle RA, Rajkumar SV. Multiple myeloma. Blood 2008; 111:2962-2972.

2. Richardson PG, Barlogie B, Berenson J, et al. A phase 2 study of bortezomib in relapsed, refractory myeloma. $\mathrm{N}$ Engl J Med 2003;348:2609-2617.

3. Chen D, Frezza M, Schmitt S, Kanwar J, Dou QP. Bortezomib as the first proteasome inhibitor anticancer drug: current status and future perspectives. Curr Cancer Drug 
Targets 2011;11:239-253.

4. San Miguel JF, Schlag R, Khuageva NK, et al. Bortezomib plus melphalan and prednisone for initial treatment of multiple myeloma. N Engl J Med 2008;359:906-917.

5. Mateos MV, Richardson PG, Schlag R, et al. Bortezomib plus melphalan and prednisone compared with melphalan and prednisone in previously untreated multiple myeloma: updated follow-up and impact of subsequent therapy in the phase III VISTA trial. J Clin Oncol 2010;28:2259-2266.

6. Mateos MV, Richardson PG, Dimopoulos MA, et al. Effect of cumulative bortezomib dose on survival in multiple myeloma patients receiving bortezomib-melphalan-prednisone in the phase III VISTA study. Am J Hematol 2015;90:314-319.

7. Kim MK, Kim K, Min CK, et al. A prospective, open-label, multicenter, observational study to evaluate the efficacy and safety of bortezomib-melphalan-prednisone as initial treatment for autologous stem cell transplantation-ineligible patients with multiple myeloma. Oncotarget 2017;8:37605-37618.

8. Richardson PG, Briemberg H, Jagannath S, et al. Frequency, characteristics, and reversibility of peripheral neuropathy during treatment of advanced multiple myeloma with bortezomib. J Clin Oncol 2006;24:3113-3120.

9. Palumbo A, Avet-Loiseau H, Oliva S, et al. Revised International Staging System for multiple myeloma: a report from International Myeloma Working Group. J Clin On- col 2015;33:2863-2869.

10. Chng WJ, Dispenzieri A, Chim CS, et al. IMWG consensus on risk stratification in multiple myeloma. Leukemia 2014;28:269-277.

11. Durie BG, Harousseau JL, Miguel JS, et al. International uniform response criteria for multiple myeloma. Leukemia 2006;20:1467-1473.

12. Richardson PG, Sonneveld P, Schuster MW, et al. Bortezomib or high-dose dexamethasone for relapsed multiple myeloma. N Engl J Med 2005;352:2487-2498.

13. Ogawa Y, Suzuki K, Sakai A, et al. Phase I/II study of bortezomib-melphalan-prednisolone for previously untreated Japanese patients with multiple myeloma. Cancer Sci 2013;104:912-919.

14. Niesvizky R, Flinn IW, Rifkin R, et al. Community-based phase IIIB trial of three UPFRONT bortezomib-based myeloma regimens. J Clin Oncol 2015;33:3921-3929.

15. Blimark C, Holmberg E, Mellqvist UH, et al. Multiple myeloma and infections: a population-based study on 9253 multiple myeloma patients. Haematologica 2015;100:107113.

16. Augustson BM, Begum G, Dunn JA, et al. Early mortality after diagnosis of multiple myeloma: analysis of patients entered onto the United Kingdom Medical Research Council trials between 1980 and 2002: Medical Research Council Adult Leukaemia Working Party. J Clin Oncol 2005;23:9219-9226. 